\title{
Influence of rainfall patterns on the efficiency of first flush storage tanks
}

\author{
S. Mambretti \& U. Sanfilippo \\ DIIAR, Politecnico di Milano, Italy
}

\begin{abstract}
The environmental impact caused by urban drainage overflows is related to both water quantity and water quality. About water quality, it has been widely recognized that usually catchment runoff is much more polluted during the initial part of a rainfall event, because of the deposits built up in the antecedent dry periods on the catchment surface and in the combined sewer conduits which are washed off in wet weather. To the aim of reducing such a first foul flush impact on the receiving water bodies, as the capacity of wastewater treatment plants is usually limited to flow rates not larger than 3-5 times the mean wastewater flow, a traditional structural work is the construction of storage tanks positioned immediately upstream the main sewer overflows of the network. Nevertheless, it is very difficult to design their volume, because the pattern of rainfall events has a relevant influence on the pollutograph, and synthetic hyetographs seem not to be adequately representative.

This paper describes a check of the performances, in terms of reduction of the pollution discharged by the overflows to the receiving water bodies, of first foul flush storage tanks designed using synthetic hyetographs with different patterns (rectangular and Chicago). Such a check is carried out numerically through a comparison with the results given by the simulation of a historical rainfall series.

The results show that synthetic hyetographs are able to represent convectivetype events, but not stratiform events. Therefore, in general, a proper design of first flush storage tanks should be carried out through historical series simulation, which is by far more complex to obtain but which ensures the correct representation of the influence of rainfall patterns, including also minor events which are very important from the point of view of water quality.

Keywords: sewer overflows, environmental impact, first foul flush, storage tanks, synthetic hyetographs.
\end{abstract}




\section{Introduction}

Water pollution spoils the water we use in our homes, the rivers and streams we use for recreation, the water needed for business, and harms all life forms, including what we eat. Therefore, plans for the reduction of pollution and the restoration of natural sustainable conditions are vital.

Because of the limits of the treatment plants, it is often necessary to overflow into rivers or lakes part of the discharge collected by the combined sewer during large events. In order to reduce discharged pollution, the use of first foul tanks is prescribed. These tanks aim to collect the discharge generated from the "first" rainfall, that washes the catchment and therefore is most polluted [1].

The mechanism of pollution accumulation during dry weather can be described by a number of models. Generally speaking, all models work under the hypotheses that the mass of pollutants increases during dry weather until a maximum is reached. In this paper the method developed by Huber and Dickinson [2] is considered; different Authors investigated the values of the parameters that rule such a model $[3,4]$. On the other hand, during a rainfall event the built up pollutants are washed off; while the qualitative description is quite easy (the more intense is the rainfall, the more pollution is washed off), several investigations have been carried out in order to estimate the parameters of the phenomenon [5-6].

Following these results, the design of such tanks is based on the idea that removing the first part of the hydrograph implies the removal of the most of the pollution mass [7]; following this idea, these structures are normally designed in order to store the first 2.5-5.0 millimetres of rain [1].

This paper reports a comparison between the results obtained through single synthetic events methods (based on design hyetographs, uniform or Chicago) and the series of recorded events from which those synthetic single events are derived. It is shown that both the use of synthetic events and the hypotheses of single events bring to wrong evaluations of the necessary size of the tanks.

\section{Model of the first foul tanks}

In this paper, only tanks of capture kind are considered, in order to collect exactly the first assigned rainfall volume and its pollution loads. Therefore, these tanks should work as shown in figure 1. During the dry weather the storage volume is empty; when rainfall starts, weir 2 is closed and weir 1 open, so the runoff is stored in the tank. When the tank is full, weir 1 closes and weir 2 opens, and therefore the incoming runoff is directly discharged to the receiving body. At the end of the event, pumps start to empty the storage volume.

Dimensions of the tanks are assumed to be equal to $25 \mathrm{~m}^{3} / \mathrm{ha}$ and $50 \mathrm{~m}^{3} / \mathrm{ha}$ (i.e. able to remove respectively the first $2.5 \mathrm{~mm}$ and the first $5.0 \mathrm{~mm}$ of rainfall).

The catchment runoff is computed considering an ideal catchment with area equal to 1 hectare and runoff coefficient equal to 1 ; in particular, it is assumed a 


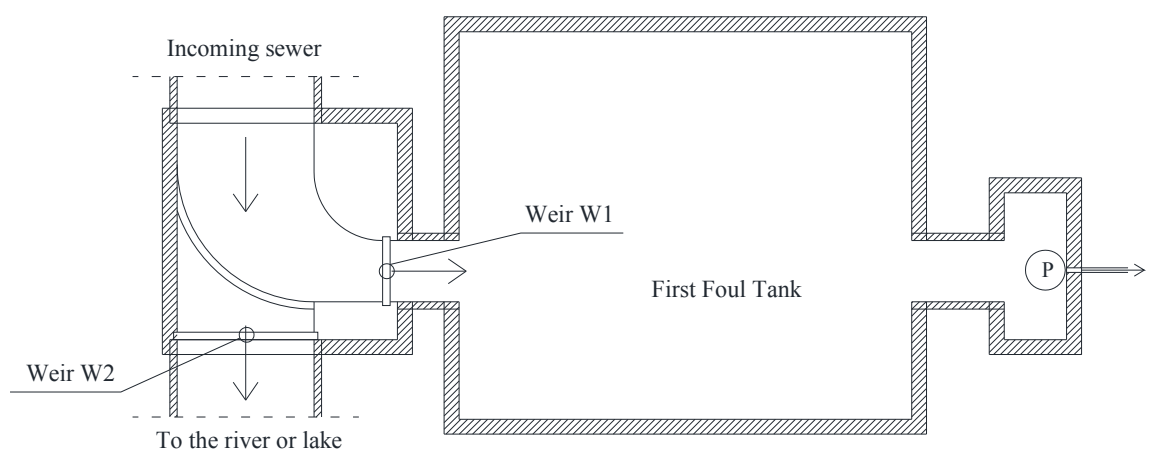

Figure 1: Plan of a first foul tank of capture kind, able to intercept exactly the assigned volume of rainfall.

linear time-area curve with the values of the concentration time chosen equal to $15,30,45$ and 60 minutes, which are well representative of an urban catchment.

In addition to the catchment runoff, to model the effect of pollutants from the catchment, the first step is modelling the accumulation of pollutants on the catchment. To this end, the Huber and Dickinson model [8] is applied:

$$
M_{a}(t)=\frac{A c c}{D i s p} \cdot\left(1-e^{-D i s p \cdot t}\right)
$$

where $M_{a}(t)[\mathrm{kg} / \mathrm{ha}]$ is the partial mass built up on the catchment at time $t[d]$; $A c c$ is the accumulation coefficient $[\mathrm{kg} /(\mathrm{ha} \mathrm{d})]$ related to the type of pollutant; Disp is the decay (also called dispersion) coefficient $\left[\mathrm{d}^{-1}\right]$ related to the wind and traffic effects. The values of the parameters are taken from literature and in accordance with those used in a previous work [1] for comparison and consistency, thus assuming $A c c=6$ and Disp $=0.2$.

The second step in the modelling process is the determination of the mass of pollutants washed off during a rainfall event. To this end, the SWMM model is applied [8]:

$$
M_{d}(t+\Delta t)-M_{d}(t)=M_{a}(t) \cdot\left(1-\exp \left(-A r r a \cdot i(t)^{\text {Wash } \cdot \Delta t}\right)\right.
$$

where $M_{d}(t)$ is the mass of pollutants washed off at time $t[\mathrm{~kg} / \mathrm{ha}] ; M_{a}(t)$ is the total mass on the catchment at time $t[\mathrm{~kg} / \mathrm{ha}] ;$ Arra is the washoff coefficient $\left[\right.$ length $\left.^{- \text {Wash }}{ }^{\text {time }}{ }^{(\text {Wash }-1)}\right]$; Wash is a dimensionless numerical parameter; $\Delta t$ is the time step [hours]; $i(t)$ is the mean precipitation intensity in the fixed time step [length time $\left.^{-1}\right]$. The values assumed by the parameters Arra and Wash depend on various factors such as the kind of substance washed away, the precipitation intensity and the shape of the catchment. Their variability is discussed in the literature, but in the present paper they are selected again in accordance with the already mentioned previous work [1], thus: $M_{a}=30 ;$ Arra $=0.00057$; Wash $=3$. 


\section{Design through single events}

In this paper, synthetic events are generated with rectangular and Chicago patterns, because they are those normally adopted for the design of the sewer systems. Simulations with rectangular hyetographs are carried out using durations equal to $5,10,15,20,30,45$ and 60 minutes. For the Chicago hyetographs, durations of 70,120 and 180 minutes are chosen, with peak position at $0.4 \cdot \vartheta$, being $\vartheta$ the duration of the rainfall.

As for the comparisons the historical series recorded by Milan - Via Monviso raingauge is taken as an example, the related DDF/IDF (Depth-DurationFrequency/Intensity-Duration-Frequency) curves for the same series are expressed in the form:

$$
i(\vartheta)=\frac{A(T)}{(\vartheta+B(T))^{C(T)}}
$$

where $i$ is the expected intensity for a given duration $\vartheta$ and return period $T ; A, B$ and $C$ are parameters carried out by regression.

Return periods and related parameters are reported in table 1.

Table 1: $\quad$ Parameters of the $I D F$ curves.

\begin{tabular}{|c|c|c|c|}
\hline$T$ & $A$ & $B$ & $C$ \\
\hline$<1$ & 67.73 & 1.046 & 2.04 \\
2 & 38.59 & 0.176 & 0.82 \\
5 & 56.06 & 0.202 & 0.85 \\
\hline
\end{tabular}

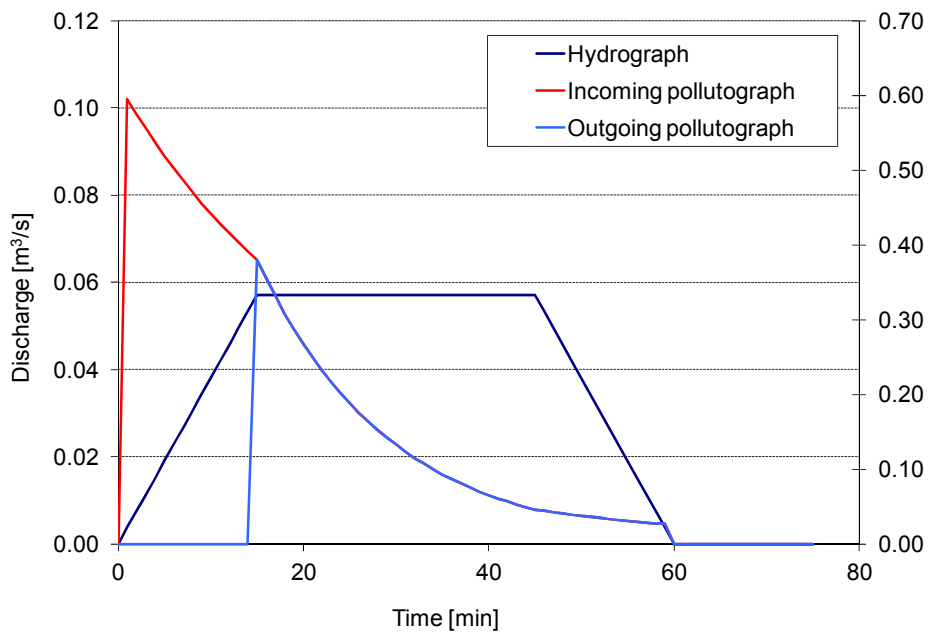

Figure 2: Hydrograph and pollutograph for $T<1$ year, duration of uniform hyetograph equal to 45 minutes and time of concentration equal to 15 minutes. 
Curves related to the return period less than 1 year are computed dividing by 2 data intensity with 2 years return period. Although this may seem quite a rough evaluation, as the distribution is logarithmic the obtained events have a very small return period, which is adequate in case of water quality problems.

Figure 2 shows the effect of a first foul tank on a hydrograph caused by a uniform rainfall. As it can be seen, the larger part of the pollution is retained in the tank, which effect is therefore significant.

The efficiency of the tank in terms of percentage of the removed pollutant mass is defined as:

$$
\eta=\frac{m_{\text {in }}-m_{\text {out }}}{m_{\text {in }}}
$$

The values of the efficiency obtained through the numerical simulations with a return period less than 1 year are summarized in table 2 . As mentioned, events with a low return period are the most important from the point of view of the pollution, because higher rainfall events imply higher dilution.

As can be seen, the efficiency of the tanks decreases when the rainfall duration increases. Moreover, for the Chicago hyetograph, the efficiency of these structures is even lower.

Table 2: $\quad$ Efficiency $\eta$ of the tanks for rainfall events with $T<1$ year.

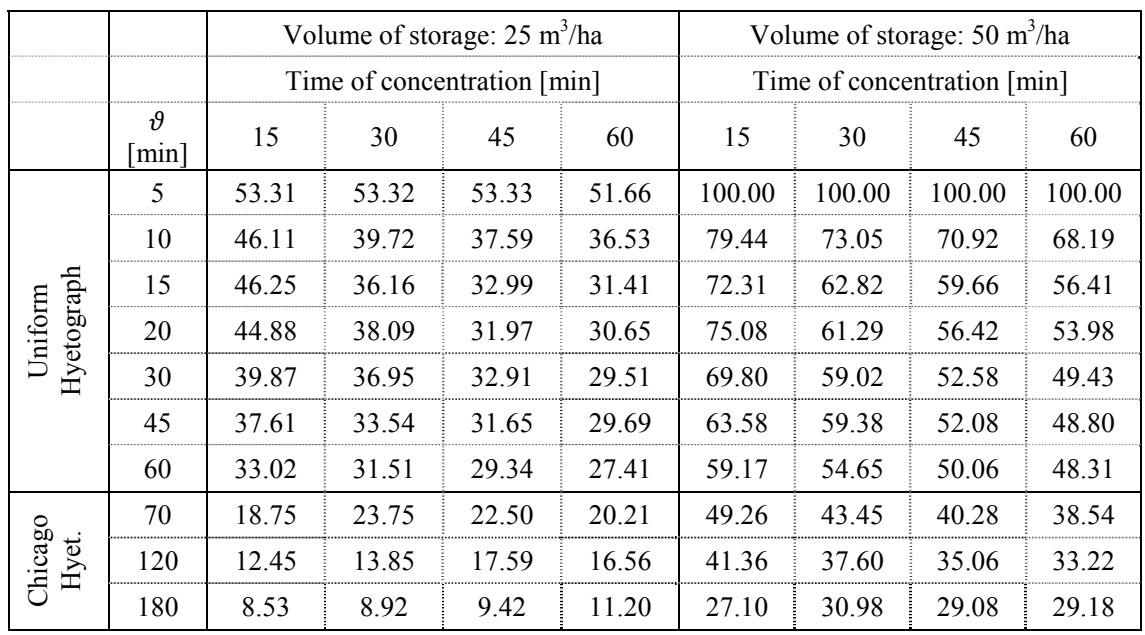

\section{Design through a rainfall series}

A similar procedure of simulation can be applied to a series of real events. In particular, an average year (1985) for the Milan - Via Monviso series has been selected for simulations. Rainfalls have been recorded with a tipping bucket raingauge and they have been divided in a number of events according to the criteria that the dry weather (no tips) between two consecutive events must be 
equal or larger than 4 days. A so large inter-event time value has been selected because this is the maximum time required by Regional Laws in Italy to empty the first foul flush storage tanks in order to restore their capacity before following event. In this way, 18 events have been identified; their main characteristics are reported in table 3.

The effects of the tanks are shown in the figures 3 and 4 for the two volumes $\left(25 \mathrm{~m}^{3} /\right.$ ha and $50 \mathrm{~m}^{3} / \mathrm{ha}$ ) and for time of concentration equal to 15 minutes. Results for time of concentration equal to 60 minutes are qualitatively similar. It must be remarked that for summer events (mainly convective in Northern Italy) tanks have a larger efficiency, while for winter events (mainly stratiform in Northern Italy) the effect of the tanks is negligible. Moreover, as it can be seen in figure 5, if the rainfall intensity is low, the first foul flush is a significantly less relevant phenomenon and the pollution is washed off gradually along the whole event although still as a function of rainfall intensity.

\section{Single event and continuous analysis}

So far, simulations of first foul tanks have been carried out dividing the historical series into a number of events, according to the criteria of a minimum interevent-time equal to 4 days, and each of them has been simulated independently. However, it must be noted that this method implies that at the beginning of the event the mass of pollutants on the catchment has already reached its maximum.

Table 3: Characteristics of the 18 rainfall events defined by an inter-eventtime of at least 4 days for the average year 1985 of Milano - Via Monviso raingauge.

\begin{tabular}{|c|c|c|}
\hline Event & $\begin{array}{c}\text { Total depth } \\
{[\mathrm{mm}]}\end{array}$ & $\begin{array}{c}\text { Maximum intensity } \\
{[\mathrm{mm} / \mathrm{h}]}\end{array}$ \\
\hline 01 & 90.0 & 6.0 \\
\hline 02 & 3.2 & 2.2 \\
\hline 03 & 161.0 & 8.9 \\
\hline 04 & 47.8 & 8.0 \\
\hline 05 & 50.6 & 26.4 \\
\hline 06 & 181.2 & 38.4 \\
\hline 07 & 86.2 & 129.6 \\
\hline 08 & 31.4 & 8.8 \\
\hline 09 & 1.8 & 5.3 \\
\hline 10 & 13.6 & 24.0 \\
\hline 11 & 39.0 & 22.1 \\
\hline 12 & 10.4 & 67.2 \\
\hline 13 & 43.2 & 60.0 \\
\hline 14 & 35.2 & 55.2 \\
\hline 15 & 68.8 & 7.4 \\
\hline 16 & 74.6 & 3.8 \\
\hline 17 & 15.6 & 3.1 \\
\hline 18 & 49.0 & 10.6 \\
\hline
\end{tabular}




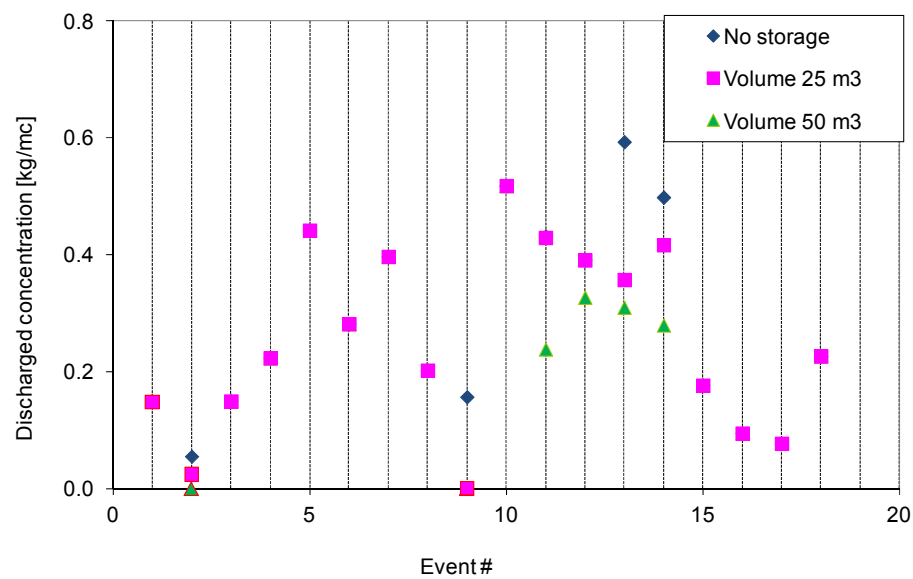

Figure 3: Comparison between discharged concentrations with and without first foul tanks $(T c=15$ minutes $)$ for the considered historical rainfall series (year: 1985).

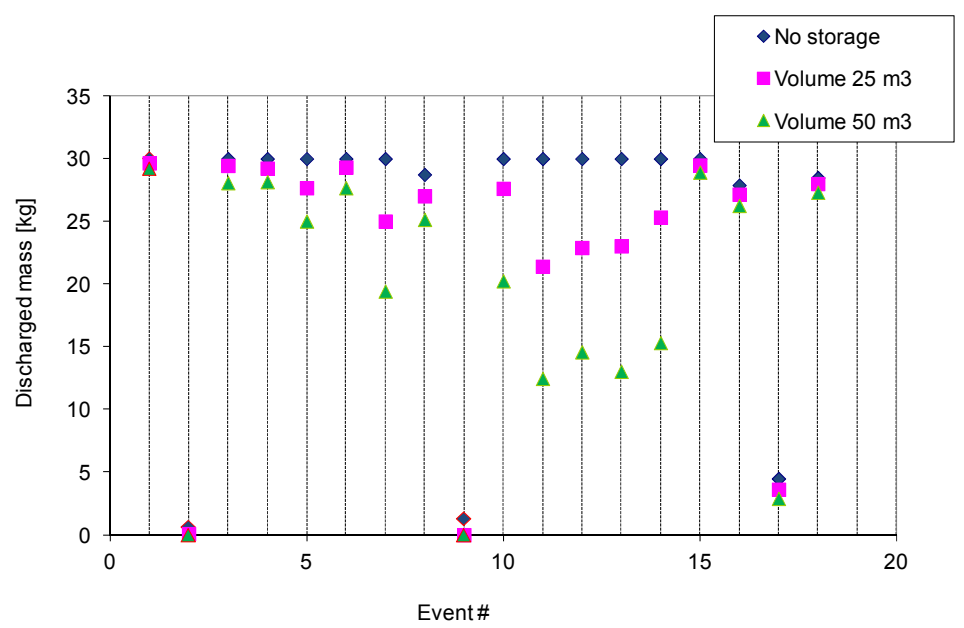

Figure 4: Comparison between discharged masses with and without first foul tanks ( $\mathrm{Tc}=15$ minutes) for the considered historical rainfall series (year: 1985).

On the other hand, given the mentioned definition of "event", each single event can be very long and may also contain quite long periods of dry weather (although shorter than four days) sometimes just interrupted by very low intensity rainfalls. In figure 6 the mass cumulated during an average year is shown: as it can be seen, only in few moments the hypothesis that the built up mass is close to the maximum is verified. 
In figure 7 the pollutographs obtained for one specific event are shown; simulations have been performed on the one hand with single event hypotheses and on the other hand with continuous simulation. As it can be seen, the maximum peak of the pollutograph is lower when the simulation is carried out continuously, because of the smaller amount of the initial mass; however, in the

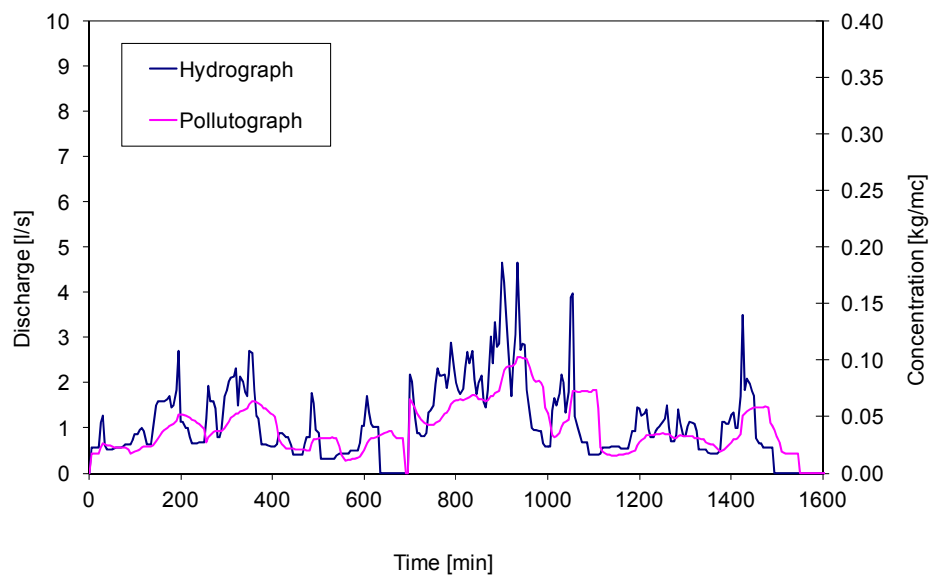

Figure 5: Event 04 of the considered historical series (year 1985): computed hydrograph and pollutograph outflowing from a catchment with $T c$ $=60 \mathrm{~min}$.

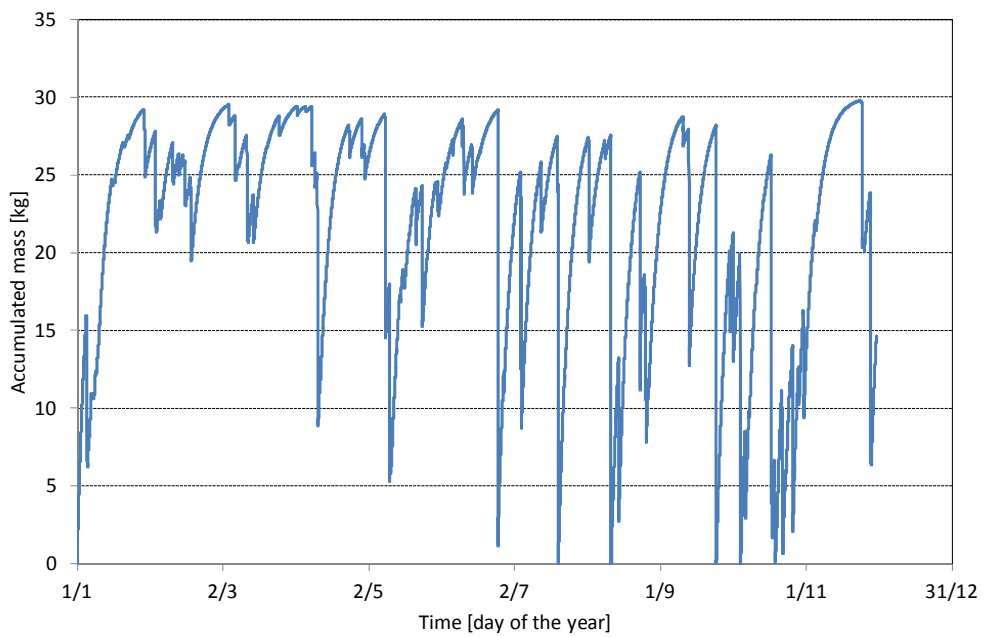

Figure 6: Accumulated mass during the average year (1985) of the considered historical series, computed through a continuous simulation. 


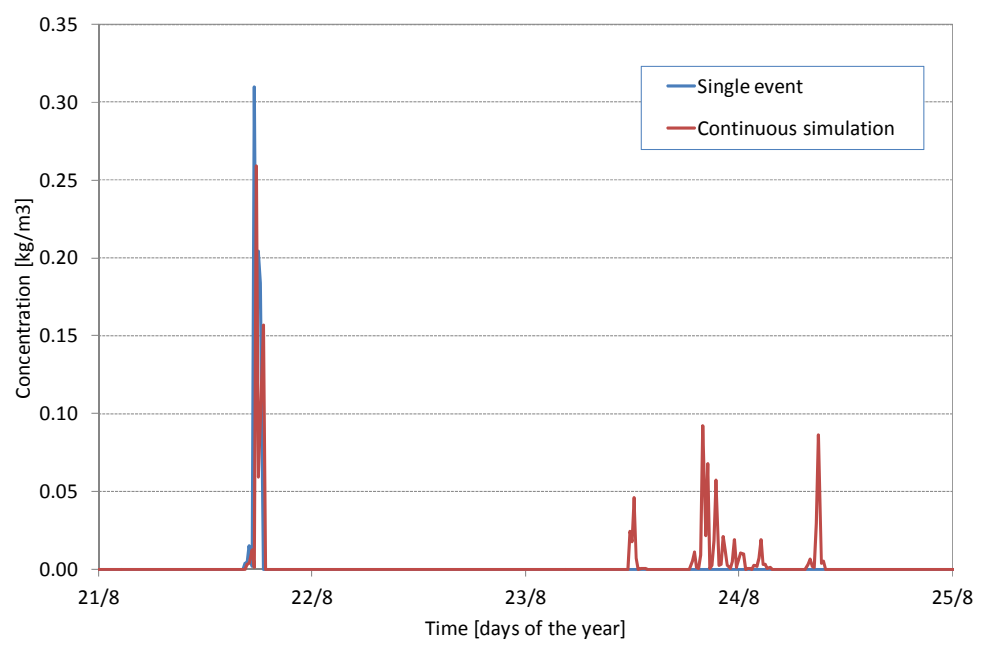

Figure 7: Computed pollutograph for the same event: single event simulation versus continuous simulation.

single event simulation the pollutograph is terminated after the first peak, while in the continuous simulation a number of subsequent peaks of pollution are visible also towards the end of the event.

On the whole, it can be seen that the first foul flush is significant when the simulation under the single event hypotheses is performed, and even more when a synthetic hyetograph is used; this importance fades when considering real events and becomes even less important when these real events are simulated in series.

\section{Conclusions}

Water pollution is a problem of increasing importance, and its reduction, together with plans for the restoration of natural sustainable conditions, is vital.

In order to reduce discharged pollution, the use of first foul tanks is often prescribed. These tanks aim to collect the discharge generated from the "first" rainfall, that washes off the pollution from the catchment and surfaces.

In this paper the design prescribed by Regional Laws in Italy, that rule the first $2.5-5.0 \mathrm{~mm}$ of rainfall have to be collected and treated, is checked by means of design hyetographs (rectangular and Chicago), with single events extracted by an historical series, and with the whole series itself.

It is shown that the hypotheses of single events bring to wrong evaluations of the necessary size of the storage volumes in the tanks. Therefore, simulations should be performed using the whole real series in order to gather a realistic picture of the phenomenon. It is also pointed out that the tanks designed as prescribed may be quite ineffective to collect the most polluted rainfalls, especially in case of events with either late peak or low mean rainfall intensities. 
The research will continue in order to check the effectiveness of these tanks in collecting deposited material on the bottom of the pipes; a further topic which deserves deeper investigation is the design of efficient first foul tanks, to improve the efficiency of those here described keeping into account the influence of the values of the build-up and wash-off parameters, of the rainfall regime and of the chosen minimum inter-event-time.

\section{References}

[1] Albizzati Mantegazza S., Gallina A., Mambretti S., Lewis C. (2010) "River Quality: a comparison of the normative criteria and dynamic modelling methods of evaluating CSO." Urban Water Journal, Vol. 7, No. 3, pp. 211216.

[2] Huber W.C., Dickinson R.E. (1988) Storm Water Management Model: User's Manual, USEPA/600/3-88/01a, EPA Athens, Georgia.

[3] Alley W.M., Smith P.E. (1981) "Estimation of accumulation parameters for urban runoff quality modelling" Water Resources Research, vol. 17, n. 6, pp. 1657-1664.

[4] Bujon G., Herremans L. (1990) "FLUPOL. Modèle de prévision des débits et des flux polluants en réseaux d'assainissement par temps de pluie: calage et validation." La Houille Blanche, n. 2.

[5] Nakamura E. (1984) "Factors affecting stormwater quality decay coefficient" Proc. of the Third ICUSD, vol. 3, Goteborg, Sweden, pp. 979988.

[6] Sueishi T., Morioka T., Kawamoto S. (1984) "Comparison among models for stormwater and pollutant runoff in view of their reliability, sensitivity and applicability" Proc. of the Third ICUSD, vol. 3, Goteborg, Sweden, pp. 605-614.

[7] Maglionico M., Pollicino F. (2004) "Experimental analysis of the build-up of pollutants on an urban road surface" Int. Conf. Novatech, Lyon, France, 6-10 June.

[8] Huber W.C., Dickinson R.E. (1988) Storm Water Management Model: User's Manual, USEPA/600/3-88/01a, EPA Athens, Georgia. 